Veritas Et Scientia

Vol. $7, N^{\circ} 2,916-924$

Julio - Diciembre del 2018.

ISSN Edición Online: 2617-0639

https://doi.org/10.47796/ves.v7i2.68

\title{
INCIDENCIA DE LA ESTRATEGIA DIDÁCTICA DE RESOLUCION DE PROBLEMAS EN EL APRENDIZAJE SIGNIFICATIVO DE MATEMÁTICA IV, INGENIERIA CIVIL EN LA UNIVERSIDAD PRIVADA DE TACNA, 2014
}

\author{
INCIDENCE OF THE DIDACTIC STRATEGY OF RESOLUTION OF PROBLEMS IN THE SIGNIFICANT \\ LEARNING OF MATHEMATICS IV, CIVIL ENGINEERING AT THE PRIVATE UNIVERSITY OF TACNA, \\ 2014.
}

Arcadio Atencio Vargas ${ }^{1}$

Presentado:07/04/2018

Aceptado: 07/05/2018

Publicado online:27/12/2018

\section{RESUMEN}

\begin{abstract}
La presente investigación tuvo como objetivo determinar la incidencia de la aplicación de la estrategia de resolución de problemas en los niveles de logro de aprendizajes significativos en el curso de matemática IV, en la carrera profesional de Ingeniería Civil, en el semestre académico 2014-I en la Universidad Privada de Tacna. De tipo aplicada, con un diseño de investigación cuasi experimental. La población de estudio estuvo constituida por 467 estudiantes matriculados en los cursos de matemática del plan de estudios de la carrera profesional de Ingeniería Civil. La muestra estaba compuesta por 45 estudiantes distribuidos en dos grupos, uno de control y el otro experimental. Los grupos de estudiantes fueron tomados en forma no aleatoria, y los tamaños fueron de 19 y 26 estudiantes respectivamente. La técnica de recolección de datos fue el examen y encuesta, con pruebas de entrada y salida en ambos grupos, cuestionario de percepción de la aplicación de la estrategia. Los resultados demostraron que la aplicación de la estrategia de resolución de problemas, en el curso de matemática IV, permitió elevar el nivel de logro de los aprendizajes significativos, de insuficiente (100\%) al nivel de logro de muy bueno (69\%) y sobresaliente (15\%), haciendo total de $84 \%$ mayor a muy bueno, en los estudiantes del cuarto ciclo de la carrera profesional de Ingeniería Civil. En conclusión, se logró comprobar que la aplicación de la estrategia de resolución de problemas, tiene alta incidencia en el resultado de los aprendizajes significativos, ello se evidencia en los resultados de la prueba de salida, en los estudiantes del grupo experimental en el curso de matemática IV, alcanzando el nivel de muy bueno.
\end{abstract}

\footnotetext{
${ }^{1}$ Doctor en Educación con mención en Gestión Educativa.
} 
Palabras claves: Resolución de problemas, aprendizaje significativo, calidad de la enseñanza.

\begin{abstract}
The objective of this research was to determine the incidence of the application of the problem solving strategy in the levels of achievement of significant learning in the Mathematics IV course, in the Civil Engineering professional career, in the academic semester 2014-I in Private University of Tacna. Of applied type, with a quasi-experimental research design. The study population consisted of 467 students enrolled in the mathematics courses of the career plan of Civil Engineering. The sample consisted of 45 students divided into two groups, one of control and the other experimental. The groups of students were taken in a non-random way, and the sizes were 19 and 26 students respectively. The technique of data collection was the examination and survey, with entrance and exit tests in both groups, questionnaire of perception of the application of the strategy. The results showed that the application of the problem solving strategy, in the Mathematics IV course, allowed to raise the level of achievement of the significant learning, from insufficient $(100 \%)$ to the level of achievement of very good $(69 \%)$ and outstanding (15\%), making a total of $84 \%$ greater than very good, in the students of the fourth cycle of the Civil Engineering career. In conclusion, it was possible to verify that the application of the problem solving strategy has a high incidence on the result of the significant learning, this is evidenced in the results of the exit test, in the students of the experimental group in the course of Mathematics IV, reaching the level of very good.
\end{abstract}

Keywords: Problem solving, meaningful learning, quality of teaching.

\title{
INTRODUCCIÓN
}

La educación superior universitaria del siglo XXI enfrenta una serie de desafíos y dificultades, como producto del entorno cambiante, la globalización y su ideal de posicionamiento efectivo en la sociedad del conocimiento. La universidad peruana no es ajena a esta realidad y para insertarse en este escenario competitivo, deben plantearse estrategias que la conduzcan a superar estos retos, los mismos que están ligados a la búsqueda de competitividad que se sostiene sobre la calidad de enseñanza y la investigación. La calidad educativa en el Perú se sostiene sobre los enfoques por competencias, del pensamiento complejo, evaluación auténtica y alineamiento constructivo. En la medida que la planificación curricular se sostenga sobre los fundamentos de los enfoques pedagógicos, la calidad de la enseñanza se encontrará asegurada. En el ámbito universitario, existen muchos desafíos relacionados con la calidad de la enseñanza. Planificar y desarrollar experiencias pedagógicas constituye una de las alternativas eficaces para mejorar la calidad de la enseñanza y de los aprendizajes. La matemática al ser parte de la ciencia formal, requiere de metodologías activas y constructivas para su aprendizaje en el aula. En ese sentido, es vital validar estrategias de enseñanza para asegurar el logro de los aprendizajes significativos. 
En la actualidad, la matemática es el soporte insustituible de los avances tecnológicos y comunicacionales de una sociedad altamente tecnificada (Álvarez y Ruiz, 2010: 227). Diversos estudios reportan que la matemática tiende a constituirse en un filtro selectivo en los distintos niveles educativos a escala mundial (Davis \& Hersh, 1998).

La evolución social, científica, técnica y económica actual requiere un aprendizaje diferente del que tradicionalmente se ha venido realizando. Los enfoques educativos han cambiado y la enseñanza tiene que cambiar. En efecto, si hace unas décadas un enfoque basado en la transmisión del conocimiento acumulado, en el que los estudiantes aprendían los fundamentos de una disciplina, parecía adecuado, quizás en estos momentos no sea suficiente. Las nuevas formas de trabajo académico exigen a los profesores tener que asumir el rol protagónico que debe cumplir el estudiante. "Como educadores matemáticos estamos interesados en que nuestros alumnos conozcan la matemática, la comprendan, la aprecien y que sean capaces de aplicarlas en la vida cotidiana y profesional". (D'Amore B. 2008: 7). El estudiante, pasó a ser el auténtico eje de la educación universitaria y el profesor un mediador o guía de dicho proceso de aprendizaje. "Este cambio supone que los profesores deben incorporar en su bagaje de conocimientos la metodología activa de enseñanza, para enfrentar con éxito la resolución de problemas matemáticos", (Piscoya, 2005). No está demás destacar que "una de las carreras con elevada dificultad en el proceso enseñanza-aprendizaje de la matemática, probablemente sea la ingeniería, puesto que esta disciplina adquiere un carácter eminentemente formativo" (Álvarez, Ruiz, 2010). No debemos olvidar que el universo se rige por leyes naturales, y la matemática es hasta ahora la mejor manera de entender estas leyes y sus relaciones. Cualquier fenómeno, es posible caracterizarlo mediante modelos matemáticos (Chamoso, 1995; Calderón, 1996).

Ausubel plantea que el aprendizaje del estudiante depende de la estructura cognitiva previa que se relaciona con la nueva información (Ausubel, 1983 :18). Respecto al aprendizaje, no solo actúa el estudiante, sino también el docente tiene la importante misión de lograr en los estudiantes aprendizajes significativos contextualizados y duraderos en el tiempo. En el campo pedagógico universitario existe una variedad de estrategias didácticas. Una de estas estrategias didácticas, es la resolución de problemas relacionados a la vida real. "La resolución de problemas aparece ahora como una actividad esencial para favorecer el cambio conceptual y metodológico." (Gil, D. 1987).

Aprender a aprender implica la capacidad de reflexionar en la forma en que se aprende y actuar en consecuencia, autorregulando el propio proceso de aprendizaje mediante el uso de estrategias flexibles y adecuadas que se transfieren y adaptan a nuevas situaciones (Diaz F., Hernández G. 2002: 234). El factor más importante es la cantidad, claridad y organización de esos conocimientos que ya tiene el estudiante al momento de iniciar nuevos aprendizajes (Bixio, C. 2001: 57). Según Schmeck (1988); Schunk (1991) "las estrategias de aprendizaje son secuencias de procedimientos o planes orientados hacia la consecución de metas de aprendizaje, mientras que los procedimientos específicos dentro de esa secuencia se denominan tácticas de aprendizaje." Según Diaz Barriga (2002), las estrategias de aprendizaje son procedimientos (conjunto de pasos, operaciones o habilidades) que un aprendiz emplea en forma consciente, controlada e intencional como instrumentos flexibles para aprender significativamente y solucionar problemas (Diaz Barriga, Castañeda y Lule, 1986). Según Stanic y Kilpatrick (1988), "Ios problemas han ocupado un lugar central en el currículo matemático desde la antigüedad, pero la resolución de problemas, no". 
Las estrategias de enseñanza son procedimientos que el agente de enseñanza utiliza en forma reflexiva y flexible para promover el logro de aprendizajes significativos en los estudiantes (Mayer, 1984; Shuell, 1998; West, Farmer y Wolff, 1991. En ese mismo entender, las estrategias de enseñanza son medios o recursos para prestar la ayuda pedagógica (Diaz F., Hernández G. 2002: 141). Jesús Toboso Picazo concluye que la habilidad para concebir un plan de resolución incide, de forma significativa, en los procesos de ejecución algorítmica que llevan a la solución final y en el rendimiento general de matemática. Estos datos vienen a corroborar, en parte, las investigaciones de Sternberg y Grigorenko (1992) y Serrano (1994), que afirma que las personas que prefieren planificar obtienen mayor rendimiento en los procesos de resolución de problemas. Entendiendo la resolución de problemas como una actividad inherente al ser humano (Piaget, 1972), es trascendente tener en cuenta que la percepción de los problemas es relativa a cada persona. En nuestra realidad, tanto en educación básica regular como en la enseñanza superior, la proliferación de ejercicios en clase de matemática ha desarrollado y arraigado en los alumnos un síndrome generalizado; "lo resuelvo" o "no lo resuelvo". Debemos entender ante esto que todo ser humano es activo procesador de la experiencia mediante el complejo sistema en el que la información es recibida, transformada, acumulada, recuperada y utilizada". Gimeno y Pérez (1993: 54). Según Moreno, et al, (1980), la estrategia es: "tomar una o varias decisiones de manera consciente e intencional que trata de adaptarse lo mejor posible a las condiciones contextuales para lograr de manera eficaz un objetivo". Respecto a estrategia didáctica, Tobón y García, señalan que "las estrategias didácticas se conciben como construcciones lógicas pensadas para orientar el aprendizaje y la enseñanza de las competencias en los diversos niveles educativos", (Tobón, S. y García A. (2014).

\section{OBJETIVOS ESPECÍFICOS}

a) Establecer el nivel de logro del aprendizaje significativo de los estudiantes de un grupo control y experimental, antes y después de la aplicación de una estrategia didáctica de resolución de problemas, en el curso de matemática del IV ciclo de la carrera profesional de Ingeniería Civil.

b) Definir el nivel de aceptación de los estudiantes de la estrategia didáctica de resolución de problemas en el curso de matemática IV, en la carrera profesional de Ingeniería Civil.

\section{METODOLOGÍA}

Investigación aplicada, cuasi experimental con aplicación de la estrategia didáctica de resolución de problemas; que consiste en aplicar una prueba de entrada y una prueba de salida al grupo experimental y al grupo de control, para determinar la eficacia de la estrategia en el proceso de aprendizaje de los estudiantes en el curso de matemática IV, durante el semestre académico 2014 - I, en la carrera de Ingeniería Civil. La muestra estuvo constituida solamente por los estudiantes matriculados: Grupo Control, 19 estudiantes y Grupo Experimental con 26 respectivamente divididos en 2 secciones, la sección A del curso de matemática IV fue intencionalmente el grupo experimental y la sección B el grupo control con un docente diferente. Se utilizó una prueba de entrada y salida, así como un cuestionario aplicado a los estudiantes del grupo experimental sobre la percepción que tuvieron en el nivel de aceptación de la estrategia didáctica. Se trabajó con el análisis estadístico inferencial, donde los resultados fueron procesados con el cálculo de las medias y las desviaciones estándares de cada grupo de estudio, con un nivel de confianza del $95 \%$. 


\section{RESULTADOS}

El $100 \%$ de estudiantes evaluados en la prueba de entrada, se encuentran en el nivel de aprendizaje insuficiente, con calificaciones menores de 10 puntos (tabla 01) así como que los 26 estudiantes del grupo experimental (100\%) se encuentran en el nivel de aprendizaje también insuficiente (tabla 02). El bajo nivel de aprendizaje logrado por los estudiantes revela que los procesos de enseñanza y aprendizaje se encuentran en el nivel de aprendizaje insuficiente con medias de las calificaciones muy similares (tabla 03)

Tabla 1

Distribución de notas por niveles de logro de aprendizaje significativo en la prueba de entrada del grupo control

\begin{tabular}{clcc}
\hline Puntajes & \multicolumn{1}{c}{ Niveles } & $\mathbf{n}$ & $\mathbf{\%}$ \\
\hline $19-20$ & Sobresaliente & 0 & $0 \%$ \\
$17-18$ & Muy bueno & 0 & $0 \%$ \\
$14-16$ & Bueno & 0 & $0 \%$ \\
$11-13$ & Suficiente & 0 & $0 \%$ \\
$00-10$ & Insuficiente & 19 & $100 \%$ \\
\hline Total & & 19 & $100 \%$ \\
\hline
\end{tabular}

\section{Tabla 2}

Distribución de notas por niveles de logro de aprendizaje significativo en la prueba de entrada en el grupo experimental.

\begin{tabular}{clcc}
\hline Puntajes & Niveles & $\mathbf{n}$ & $\mathbf{\%}$ \\
\hline $19-20$ & Sobresaliente & 0 & $0 \%$ \\
$17-18$ & Muy bueno & 0 & $0 \%$ \\
$14-16$ & Bueno & 0 & $0 \%$ \\
$11-13$ & Suficiente & 0 & $0 \%$ \\
$00-10$ & Insuficiente & 26 & $100 \%$ \\
\hline Total & & 26 & $100 \%$ \\
\hline
\end{tabular}

Fuente: Prueba de entrada 


\section{Tabla 3}

Comparación de las medidas de tendencia central y dispersión de las calificaciones obtenidas en la prueba de entrada

\begin{tabular}{lcc}
\hline \multicolumn{1}{c}{ Estadísticos } & \multicolumn{2}{c}{ Prueba de Entrada } \\
\cline { 2 - 3 } & Grupo Control & Grupo Experimental \\
\hline Media aritmética & $\bar{X}_{c}=3.63$ & $\bar{X}_{e}=3.23$ \\
Desviación estándar & $S_{c}=0.93$ & $S e=1.62$ \\
Tamaño de muestra & $n_{c}=19$ & $n_{e}=26$ \\
\hline
\end{tabular}

Se concluye con un nivel de confianza del 95\%, que el nivel de aprendizaje obtenido en la evaluación de la prueba de entrada, por los estudiantes del grupo de control y experimental es insuficiente $(<10$ puntos) ( $p:<0.05)$.

Tabla 04

Medidas estadísticas de las calificaciones de logro de aprendizaje en la prueba de salida en los estudiantes del grupo de control y grupo experimental.

\begin{tabular}{lcc}
\hline \multicolumn{1}{c}{ Estadísticos } & \multicolumn{2}{c}{ Prueba de salida } \\
\cline { 2 - 3 } & Grupo Control & Grupo Experimental \\
\hline Media aritmética & $\bar{X}_{c}=11.34$ & $\bar{X}_{e}=17.31$ \\
Desviación estándar & $S_{c}=3.07$ & $S_{e}=1.54$ \\
Tamaño de muestra & $n_{c}=19$ & $n_{e}=26$ \\
\hline & & \\
\end{tabular}

En la tabla 04 se observa que la media aritmética obtenido por los estudiantes del grupo experimental, con la aplicación de la estrategia, es muy superior (17.31) al promedio obtenido en la prueba de salida del grupo de control (11.34). Las diferencias se deben fundamentalmente a la incidencia de la estrategia de solución de problemas en el aprendizaje de ecuaciones diferenciales en el modelamiento y solución de situaciones problemáticas concretas de las diferentes áreas del conocimiento relacionado con la carrera profesional de los estudiantes.

Con respecto al análisis de los niveles de aceptación de los estudiantes respecto a la estrategia didáctica de resolución de problemas, (Fig. 1), el 57.7\% aceptan totalmente la estrategia, el 
38.5\% la aceptan regularmente y el 3.8\% (un estudiante), muestra una baja aceptación. No debemos olvidar que la percepción y actitud expresado en una encuesta por parte de los estudiantes es relativa, más aún si se trata de un curso de matemática.

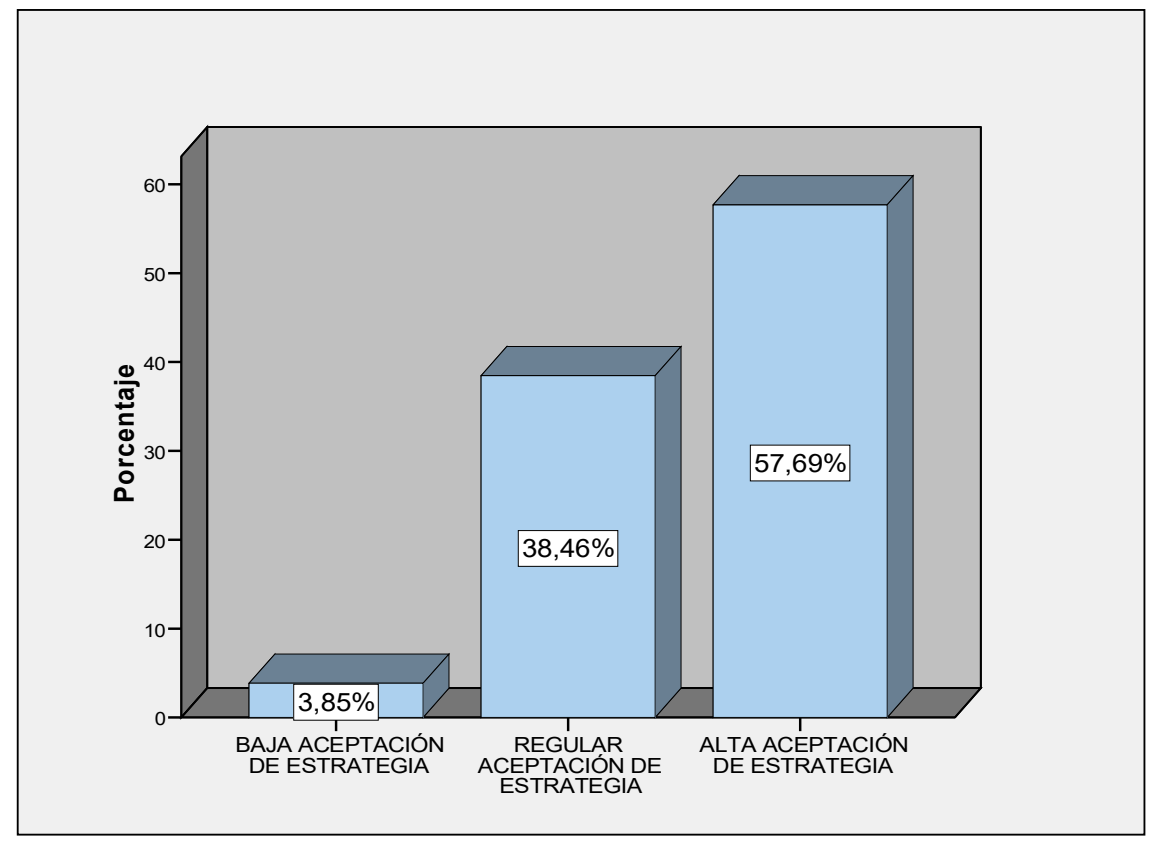

Figura 1 Niveles de aceptación respecto a la estrategia didáctica de resolución de problemas.

\section{DISCUSIÓN}

En nuestro estudio, la aplicación de la estrategia didáctica de resolución de problemas tiene alta incidencia en el nivel de aprendizaje significativo de los estudiantes en el curso de matemática del IV ciclo en la carrera profesional de Ingeniería Civil, en la Universidad Privada de Tacna, 2014-I. En promedio el 96\% de los estudiantes están de acuerdo con la aplicación de la estrategia didáctica de resolución de problemas. Es decir, la mayoría de los estudiantes han entendido que la solución de problemas es a menudo la única manera factible de probar si en realidad comprendieron significativamente Ecuaciones Diferenciales y que, además son capaces de expresar verbalmente lo aprendido.

Estos resultados guardan relación con los obtenidos por Varela P. (1992), Toboso J. (2004), Mendoza M. (2014), quienes de manera general expresan que después de la aplicación de una estrategia como la de resolución de problemas, el aprendizaje significativo en cursos de ciencias o la matemática, es muy evidente. Sin embargo, es importante destacar que Paloma Varela (1992), también controla el cambio conceptual que los estudiantes adquieren, así como las diferencias individuales y que en nuestro estudio no se tomó en cuenta, pero en el proceso de aprender a aprender con la estrategia de resolución de problemas. De acuerdo a los resultados de este estudio, la estrategia didáctica de resolución de problemas en la enseñanza de matemática a nivel superior puede ser extensivo a cualquier curso de matemática en la carrera o en la Universidad, más aún, cuando es necesario que el docente "debe hacer transitar a los estudiantes por algunas de las fases del proceso de investigación científica" 
(Ortiz O. 2006), como lo hace la estrategia que hemos empleado. La necesidad de investigación en la mayoría de nuestros estudiantes es aún insipiente; el ejemplo debemos dar los profesores.

Nuestro estudio demuestra que el aprendizaje significativo en el área de matemáticas, requiere de procesos didácticos focalizados en el desarrollo de las competencias y en la demostración de desempeños de los estudiantes. El aprendizaje tiene que ser auténtico y permitir que el estudiante pueda aplicar los conocimientos en la solución de un problema del contexto real. Permite demostrar que el aprendizaje significativo en el área de matemáticas, es un proceso que requiere estrategias de calificación que categorice a los estudiantes por niveles de rendimiento y desempeño. Evaluar sobre aprendizajes superficiales respecto de una evaluación de aprendizajes profundos, requiere de metodologías de enseñanza que asegure el desarrollo de las competencias y la mejora de los desempeños de los estudiantes. El aprendizaje para ser auténtico tiene que ser significativo y desarrollarse en escenarios reales y brindar soluciones de un problema del contexto real.

El enfoque de calificaciones de Díaz (1988) afirma que las notas deben estar ubicadas en niveles de logro de aprendizaje y escalas establecidas, que permitan la interpretación del aprendizaje alcanzado. Al respecto Biggs (2008:180) considera que existen dos modalidades básicas para la asignación de calificaciones: El modelo de medida y el modelo de los niveles. El primero está diseñado para acceder a las características estables de los individuos, con el fin de compararlos entre sí o con normas de la población general. Se ocupa de hacer juicios sobre las personas. Esta evaluación está referida a la norma. El segundo está diseñado para evaluar los cambios de rendimiento a consecuencia del aprendizaje, con el fin de comprobar si se ha aprendido algo y hasta qué punto se ha aprendido bien. Se ocupa de hacer juicios sobre la actuación. Esta evaluación está referida a criterios de desempeño. La presente investigación, permite demostrar que el aprendizaje significativo es un aprendizaje profundo, que requiere de procesos didácticos complejos de razonamiento analítico e inferencial, de tal forma que las estrategias de enseñanza y evaluación permiten construir escalas de medición y de calificación del rendimiento y desempeño. Finalmente, el aprendizaje significativo en el área de matemática es factor de fuerte incidencia en el proceso de desarrollo de competencias y de mejora de desempeños en contextos reales. Finalmente, de las diversas lecturas de investigaciones revisadas sobre esta temática concluimos que la mayoría de docentes realizan solamente indagaciones para poder cumplir con su responsabilidad de enseñar, solo se quedan en el nivel de acopio de información que se sistematiza para efectos de enseñar y lograr que los estudiantes aprendan.

El papel de la estrategia didáctica de resolución de problemas para el aprendizaje significativo, tiene como meta desafiante en el proceso de aprendizaje de la matemática, que el estudiante sea capaz de actuar en forma autónoma y autorregulada y los docentes deben poseer la capacidad de diseñar y aplicar diversas estrategias, sobre la base del dominio de conocimiento teórico y práctico de la matemática, que le permita la implementación de instrumentos y técnicas para evaluar los aprendizajes de los estudiantes. Los docentes deben de conocer y aplicar en forma correcta, los procesos didácticos.

\section{REFERENCIAS BIBLIOGRÁFICAS}

ALVAREZ Y., RUIZ M. (2010). Actitudes hacia las matemáticas en estudiantes de ingeniería en universidades autónomas venezolanas. Revista de pedagogía Vol. 31, Nº 89: Universidad Central de Venezuela. 
AUSUBEL, NOVAK, HANESIAN (1983). Psicología educativa: Un punto de vista cognoscitivo. México: Ed. Trillas.

BIGGS, J. (2006). Calidad del aprendizaje universitario. Madrid: Narcea S.A. de Ediciones.

BIXIO C. (2001). Enseñar a aprender. Rosario. Santa Fe: Homo Sapiens Ediciones.

CALDERON A. (1996). Investigación y didáctica de las matemáticas. Madrid: Narcea.

CHAMOSO J. (1995). Hacia unas nuevas matemáticas. Universidad de Salamanca.

DAVIS P. \& HERSH R. (1998). Experiencias matemáticas. Madrid: Labor.

DIAZ BARRIGA F., HERNADEZ G. (2002). Estrategias docentes para un aprendizaje significativo. 2da. Ed. México: Mc Graw Hill.

DÍAZ, E. (1988). Aprender a estudiar. Madrid: ICCE.

GIMENO, J. PEREZ GOMEZ, A. (1985). La enseñanza: su teoría y su práctica. Madrid: Akal Ediciones.

HERNÁNDEZ, R. (2010). Metodología de la investigación. Quinta edición. México: Mc GRAW HILL.

MAYER, R.E.; LARKIN, J.H. Y KADANE, J.B. (1984). A cognitive analysis of mathematical problem-solving ability. En Sternberg, R.J. (Ed.), Advances in the psychology of human intelligence. Vol. 2. Hillsdale, New Jersy: Lawrence Erlbaum

MORENO y SASTRE. (1980). Aprendizaje y construcción de conocimientos. Barcelona: Gedisa

PIAGET, J. (1977). Recherches sur l'abstration reflechissante. París: Presses Universitaires de France. En castellano, Investigaciones sobre la abstracción reflexionante. Buenos Aires: Huemul, 1979.

PISCOYA, L. H. (2005). Cuánto saben nuestros maestros. Una entrada a los diez problemas cardinales de la educación peruana. Lima: Fondo Editorial de la Universidad Nacional Mayor de San Marcos.

STERNBERG, R. J. (1982a). Razonamiento, resolución de problemas e inteligencia. En Sternberg, R.J. (Ed.), Inteligencia humana, vol. 2. Barcelona: Paidós Ibérica, 1987.

STERNBERG, R. J. (1985c). Las capacidades humanas: un enfoque desde el procesamiento de la información. Barcelona: Editorial Labor.

VARELA N. P. (1991). La resolución de problemas en la enseñanza de las ciencias. Aspectos didácticos y cognitivos. Tesis doctoral: Universidad Complutense de Madrid. 